\title{
HSM: A New Color Space used in the Processing of Color Images
}

\author{
Severino Jr, Osvaldo ${ }^{1}$ \\ Gonzaga, Adilson ${ }^{2}$
}

\begin{abstract}
Resumo: Inspired on the techniques used by painters to overlap layers of various hues of paint to create oil paintings, and also on observations of the the arrangement of Short-(S), Middle-(M), and Long-(L) wavelength-sensitive cones of the human retina for the interpretation of the colors, this paper proposes the use of the new color space called HSM to the processing of color images. To demonstrate the applicability of the HSM color space in the processing of color images, this paper proposes the pixelbased segmentation of a digital image of "human skin" or "non-skin", the sketch of the face image and the pixel-based segmentation of the trumpet flowers tree (ype). The performance of the HSM color space in the pixel-based segmentation is compared with the HSV, YCbCr and TSL color spaces while the sketch of the face image is also compared with HSV, YCbCr and TSL colors spaces and the edge detectors of the Sobel, Prewitt, Roberts, Canny and Laplacian of Gaussian methods. The results demonstrate the potential of the proposed color space.
\end{abstract}

\section{Introduction}

According to Morris [11], the notion of color in visual arts is associated to the pigment of inks used and the terms pure color, pastel color, shading and tone. The shading is the result of adding a black pigment to a pure pigment. The pastel color is the result of adding a white pigment to a pure pigment. The tone is the result of adding the black and white pigments to a pure pigment simultaneously. Thus, the definition of a color in visual arts is given by the specification of the pure, white and black pigments, so that the desired tone is achieved.

Several studies using indirect measurements have suggested that there are on average more $R$ cones than $G$ cones in the human retina, with large intersubject variability $[4,6]$. Using high-resolution adaptive-optics imaging combined with retinal densitometry, Hofer et al. [7] characterized the arrangement of Short- $(S)$, Middle- $(M)$, and Long- $(L)$ wavelengthsensitive cones in eight human foveal cone mosaics. As suggested by previous studies, they found males with normal color vision that varied in the ratio of $L$ (or $R$ ) to $M$ (or $G$ ) cones

\footnotetext{
${ }^{1}$ Department of Electrical Engineering - School of Engineering - USP, SP, ZIP Code 13566-590 \{osvaldo@fafica.br\}

${ }^{2}$ Department of Electrical Engineering - School of Engineering - USP, SP, ZIP Code 13566-590

\{agonzaga@sc.usp.br\}
} 
from 1.1:1 to 16.5:1.Morris's studies [11] suggest a (2:1) ratio for the distribution of red cones (type $R$ ) to green cones (type $G$ ) in the human retina.

Considering that the definition of a color in visual arts is given by the specification of the pure, white and black pigments, new tones emerge as new layers of pigments are added and a (2:1) ratio for the distribution of red cones (type $R$ ) to green cones (type $G$ ) in the human retina, this paper proposes the term Color Mixture and set the new HSM (Hue, Saturation and Mixture) color space [14] in the pixel-based segmentation by the color luminance of a digital image of "human skin" or "non-skin", the sketch of the face image and the pixelbased segmentation by the color luminance of the trumpet flowers tree. To demonstrate the applicability of the HSM color space in the processing of color images, the results obtained in the pixel-based segmentation is compared with the HSV, YCbCr and TSL color spaces while the sketch of the face image obtained in HSM color space is also compared with HSV, $\mathrm{YCbCr}$ and TSL colors spaces and the edge detectors of the Sobel, Prewitt, Roberts, Canny and Laplacian of Gaussian methods.

\section{HSM Color Space defined by the Color Mixture}

This paper proposes the use of the new color space HSM (Hue Saturation Mixture) defined by the value of the color mixture, as a tool in image-processing algorithms that take into account the characteristics of colors seen by the human visual system.

\subsection{Conversion from RGB color space to HSM color space}

The mixture value $m$ is the intersection of the main diagonal of the RGB cube with the plane (equation 1) which defines the vertical intensity axis of the HSM color space. The hue of the arbitrary color point of plane is determined by an angle from the arbitrary color point and red axis of RGB cube. So an angle $0^{\circ}$ from red axis designates 0 hue, and the hue increases counterclockwise from there. The saturation (distance from vertical intensity axis of HSM) is the length of the vector from the origin to the point. Note that the origin is defined by the intersection of the color plane with the vertical intensity axis (Figure 1).

$$
m=\frac{4 r+2 g+b}{7}
$$

Using the values of $(0 \leq r \leq 1),(0 \leq g \leq 1)$ and $(0 \leq b \leq 1)$ to calculate the values $h$ (equations 2-4), $s$ (equation 5) and $m$ of the HSM color space [14]. 


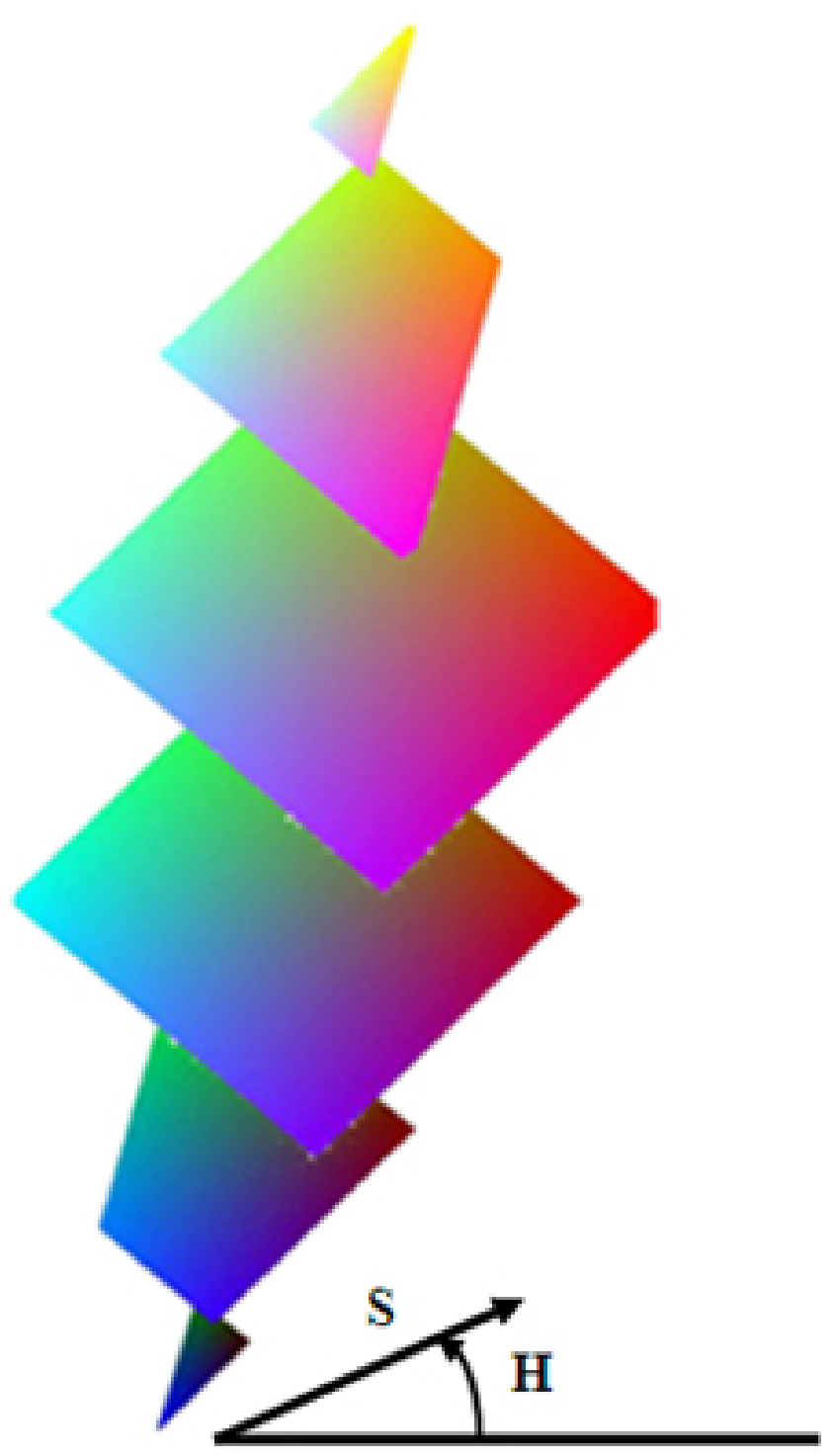

Figura 1. Definition of hue and saturation of the HSM color space. 


$$
\theta=\cos ^{-1}\left(\frac{\frac{3(r-m)-4(g-m)-4(b-m)}{\sqrt{41}}}{\sqrt{(r-m)^{2}+(g-m)^{2}+(b-m)^{2}}}\right)
$$

i.e.

$$
\begin{cases}\omega=\theta & \text { if } b \leq g \\ \omega=2 \pi-\theta & \text { if } b>g\end{cases}
$$

Thus, $h$ is defined as normalized $\omega$

$$
\begin{aligned}
& h=\frac{\omega}{2 \pi} \\
& s= \begin{cases}\frac{\sqrt{(r-m)^{2}+(g-m)^{2}+(b-m)^{2}}}{\sqrt{(0-m)^{2}+(0-m)^{2}+(7-m)^{2}}} & \text { if } 0 \leq m \leq \frac{1}{7} \\
\frac{\sqrt{(r-m)^{2}+(g-m)^{2}+(b-m)^{2}}}{\sqrt{(0-m)^{2}+\left(\frac{7 m-1}{2}-m\right)^{2}+(1-m)^{2}}} & \text { if } \frac{1}{7}<m \leq \frac{3}{7} \\
\frac{\sqrt{(r-m)^{2}+(g-m)^{2}+(b-m)^{2}}}{\sqrt{\left(\frac{7 m-3}{2}-m\right)^{2}+(1-m)^{2}+(1-m)^{2}}} & \text { if } \frac{3}{7}<m \leq \frac{1}{2} \\
\frac{\sqrt{(r-m)^{2}+(g-m)^{2}+(b-m)^{2}}}{\sqrt{\left(\frac{7 m}{4}-m\right)^{2}+(0-m)^{2}+(0-m)^{2}}} & \text { if } \frac{1}{2}<m \leq \frac{4}{7} \\
\frac{\sqrt{(r-m)^{2}+(g-m)^{2}+(b-m)^{2}}}{\sqrt{(1-m)^{2}+\left(\frac{7 m-4}{2}-m\right)^{2}+(0-m)^{2}}} & \text { if } \frac{4}{7}<m \leq \frac{6}{7} \\
\frac{\sqrt{(r-m)^{2}+(g-m)^{2}+(b-m)^{2}}}{\sqrt{(1-m)^{2}+(1-m)^{2}+((7 m-6)-m)^{2}}} & \text { if } \frac{6}{7}<m \leq 1\end{cases}
\end{aligned}
$$

\subsection{Conversion from HSM Color Space to RGB Color Space}

The conversion of the HSM space to the RGB space uses the values $(0 \leq h \leq 1)$, $(0 \leq s \leq 1)$ and $(0 \leq m \leq 1)$. One has that $r$ is calculated by equation $6, g$ is calculated by equation 7 and $b$ is calculated by equation 8 . 


$$
\begin{gathered}
r=\frac{3}{41} s \cos (h)+m-\frac{4}{861} \sqrt{\left(861 s^{2}\left(1-\cos ^{2} h\right)\right)} \\
g=\frac{\sqrt{41} s \cos (h)+23 m-19 r}{4} \\
b=\frac{11 r-9 m-\sqrt{41} s \cos (h)}{2}
\end{gathered}
$$

\section{Segmentation by the Color Luminance}

In the literature on Computer Vision, the detection of skin color is often used as a segmentation phase in biometric systems for facial recognition [8] as well as other features such as the geometry and shape of the skin.

Based on the use of skin color as a relevant characteristic for describing the image of a human face, this paper proposes to apply color luminance to segment the region of human skin and the face sketch into digital images.

\subsection{Materials and Methods}

The RGB color space has shown excellent performance in modeling and detecting regions of the human skin $[13,16,17]$.

This paper proposes the HSM color space, which was inspired on the technique that artists employ to apply layers of paint to achieve perfect colors in their works. This methodology is exploited here in terms of its ability and performance in the segmentation of the region of human skin, in the segmentation of the trumpet flowers tree and face sketch in digital images.

Considering color luminance, the proposed application was implemented in the HSM, HSV, HSL, TSL, YCbCr and RGB color spaces using MatLab version $7(R 14)$ language for the Windows XP operating system. In addition, a human skin test database was set up containing 380 RGB AR [10] database facial images with 8 bits per channel and a spatial resolution of $576 \times 768$ pixels per image, as well as 882 RGB FERET [12] database facial images with 8 bits per channel and a spatial resolution of $768 \times 512$ pixels per image, making a total of 1262 images. Initially, the human skin test database images were segmented manually 
to create the "ground truth". The trumpet tree database was also set up containing 640 RGB images with 8 bits per channel and a spatial resolution of $170 \times 113$ pixels per image captured on the web containing pink trumpet tree, golden trumpet tree and angel trumpet tree.

\subsection{Color Luminance}

In the literature, color luminance in the RGB color space corresponds to the grayscale, which is calculated by the arithmetic mean of $r, g$ and $b$ in the point (the orthogonal projection of the point with the main diagonal of the RGB cube).

The value of color luminance in other color spaces such as HSI (Hue, Saturation and Intensity) or HSL (Hue, Saturation and Lightness), HSV (Hue, Saturation and Value) and the proposed HSM (Hue, Saturation and Mixture) can be calculated by equations 9-11. In TSL (Tint, Saturation and Lightness) and YCbCr [5], the value $y$ can be calculated by equation 12. It can be deduced immediately that the luminance images generated in these color spaces will be different. Figure 2 shows the luminance images generated in this work. The values of $(0 \leq r \leq 255),(0 \leq g \leq 255)$ and $(0 \leq b \leq 255)$ are then used to generate the $i$ (HSI and $\mathrm{HSL}), v$ (HSV), $m$ (HSM) and $y$ (YCbCr and TSL) equations.

$$
\begin{gathered}
i=\frac{1}{3}(r+g+b) \\
v=\max (r, g, b) \\
m=\frac{4 r+2 g+b}{7} \\
y=0.299 r+0.587 g+0.114 b
\end{gathered}
$$

\subsection{The Human Skin Color Segmentation}

The differences of luminance in the region of the face obtained by the different color spaces must be stricty observed. So, this paper proposes a method for the pixel-based segmentation of the human skin color, called the method of difference in luminance (MDL). This 


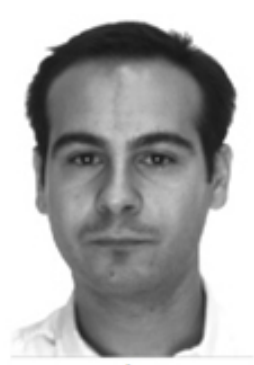

a)

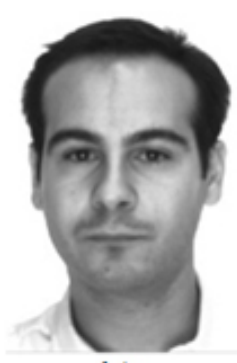

b)

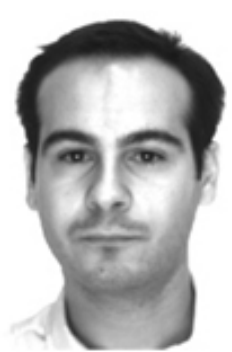

c)

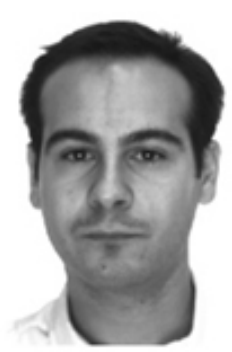

d)

Figura 2. Luminance images. a) RGB, HSI and HSL; b) HSM; c) HSV; d) TSL and YCbCr.

method calculates the difference in the luminance of the image between any color space and RGB. The pixels of the HSM image and RGB image that make the difference in luminance equal to or greater than 0.0137 is classified as a pixel of the human skin color. The pixels of the HSV image and RGB image that make the difference in luminance equal to or greater than 0.0157 is classified as a pixel of the human skin color. The pixels of the $\mathrm{YCbCr}$ image and RGB image that make the difference in luminance equal to or greater than 0.0196 is classified as a pixel of the human skin color. These values were calculated by the curve ROC (Receiver Operating Characteristic) [9] where the false match rate is igual to false non-match rate. Figure 3 illustrates the pixel-based segmentation of human skin obtained by the MDL in the HSM, HSV and YCbCr color spaces and the "ground truth" image used to calculate the false match rate and false non-match rate.

\subsection{The Face Sketch Segmentation}

In this work was observed that the HSM image where $(0 \leq M D L<0.0137)$, the HSV image where $(0 \leq M D L<0.0157)$ and the YCbCr image where $(0 \leq M D L<$ 0.0196 ) were similar to the face sketch, so the name of these new images were called "face sketch". These values were also calculated by the curve ROC where the false match rate is igual to false non-match rate. Figure 4 shows the new "face sketch" image generated by MDL in the HSM, HSV and YCbCr images compared with images obtained by the edge detectors of Sobel, Prewitt, Roberts, Laplacian of Gaussian and Canny methods implemented by MatLab (the threshold value was calculated automatically by MatLab). 

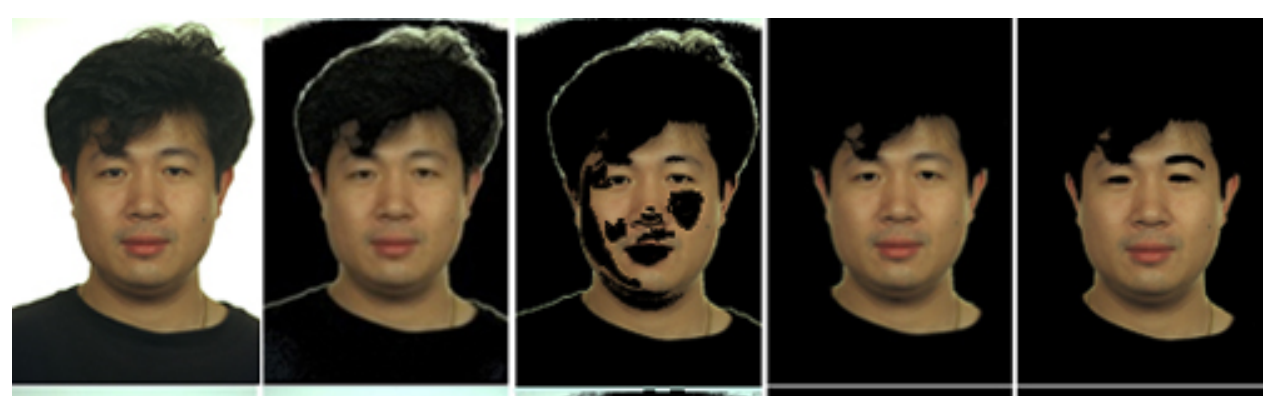

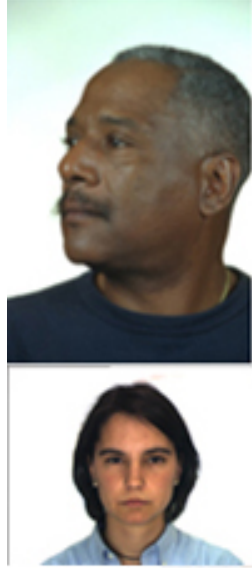

a)

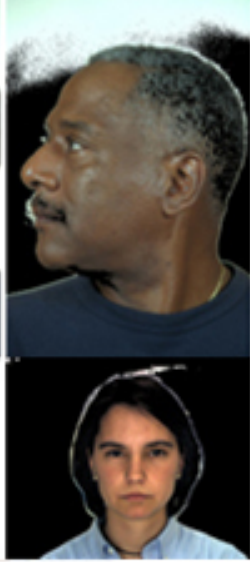

b)

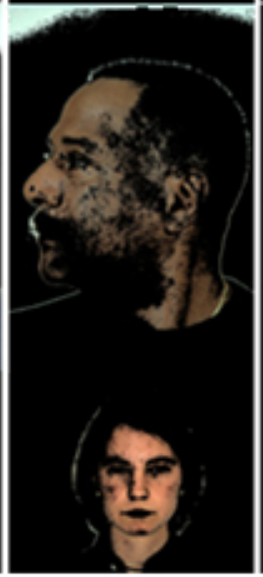

c)

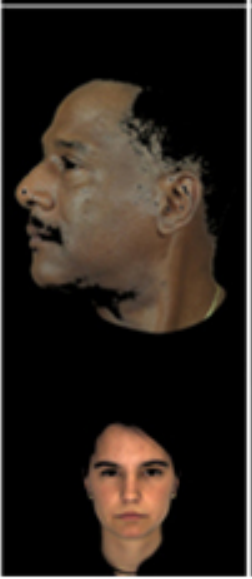

d)

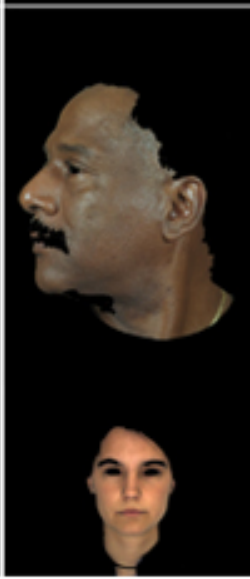

e)

Figura 3. Segmentation of Human Skin. a) Original image; b) MDL in HSV image; c) MDL in YCbCr image; d) MDL in HSM image; e) Ground truth images. 
a)

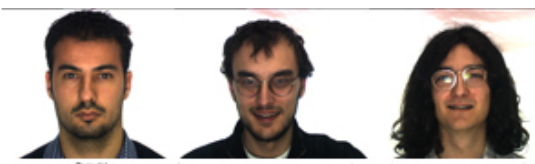

b)

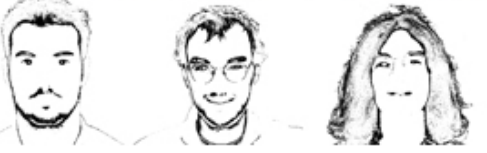

c)

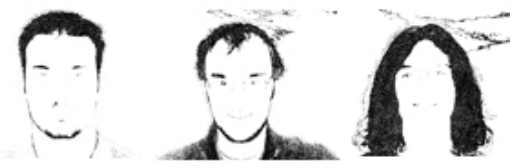

d)

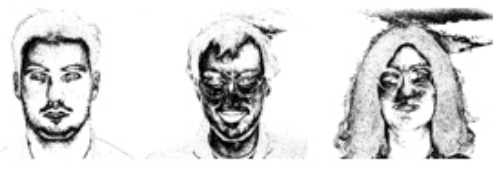

e)

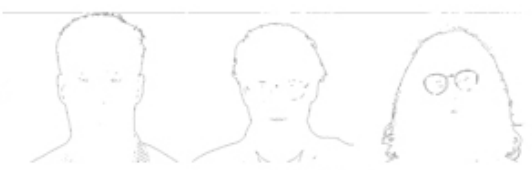

f)

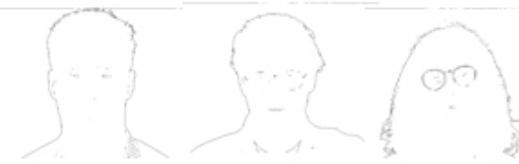

g)

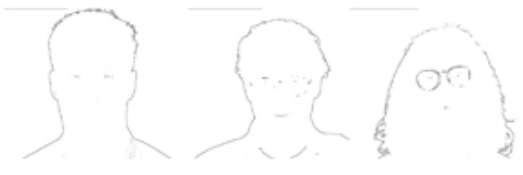

h)

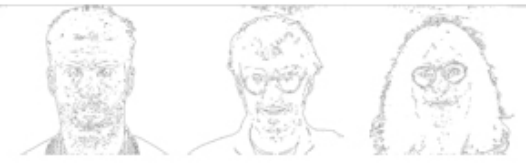

i)

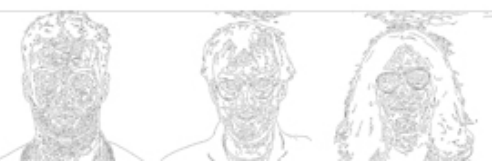

Figura 4. Face Sketch. a) Original image. b) HSM image. c) HSV image. d) YCbCr image. e) Edge detectors of Sobel method. f) Edge detectors of Prewitt method. g) Edge detectors of Roberts method. h) Edge detectors of Laplacian of Gaussian method. i) Edge detectors of Canny method. 


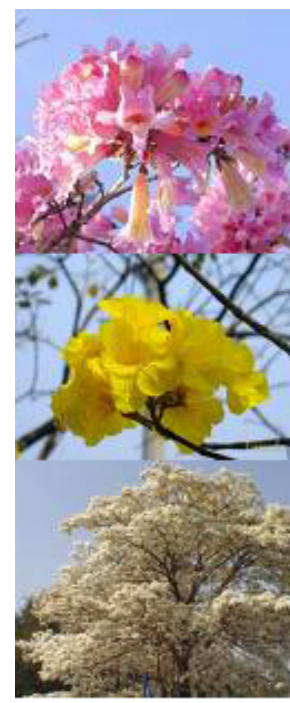

a)

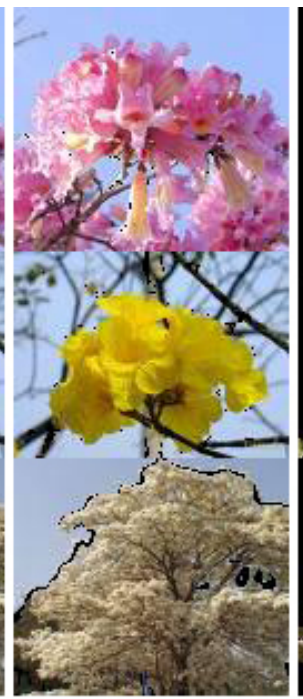

b)

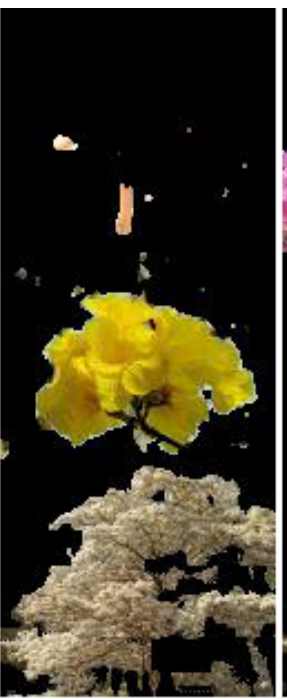

c)

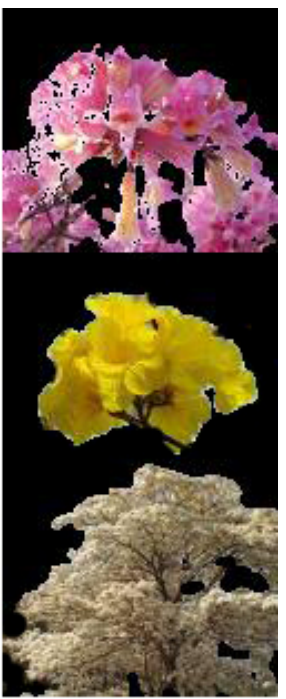

d)

Figura 5. The trumpet flower tree color (pink, golden and angel). a) Original image; b) HSV image; c) YCbCr image; d) HSM image.

\subsection{The Trumpet Flowers Tree Segmentation}

It was analyzed the performance of the MDL in the segmentation of pink trumpet flower tree, golden trumpet flower tree and angel trumpet flower tree. Figure 5 illustrates the segmentation of pink trumpet flower tree, golden trumpet flower tree and angel trumpet flower tree obtained by the $(0 \leq M D L<0.0157)$ in the HSM images, by the $(0 \leq M D L<$ $0.0157)$ in the HSV images and by the $(0 \leq M D L<0.0196)$ in the YCbCr images. These values also were calculated by the curve ROC where the false match rate is igual to false non-match rate.

\section{Results}

The Human Skin images segmented by the MDL in the HSM, HSV and YCbCr color spaces were then compared with the "ground-truth" images (obtained manually) according to the criteria listed in Table 1, which shows the performance of color spaces with respect to their overall precision and Kappa index of agreement [3]. 
HSM: A New Color Space used in the Processing of Color Images

Tabela 1. Performance index of the classifiers.

\begin{tabular}{l||c|c|c}
\hline \hline Color Spaces & HSM & HSV & YCbCr \\
\hline skin color classification rate & $98.7 \%$ & $96.4 \%$ & $52.3 \%$ \\
\hline non-skin color classification rate & $89.7 \%$ & $56.5 \%$ & $86.7 \%$ \\
\hline Overall precision & $92.2 \%$ & $67.4 \%$ & $77.3 \%$ \\
\hline Kappa index of agreement & 0.82 & 0.39 & 0.41 \\
\hline \hline
\end{tabular}

Tabela 2. Interpretation of the Kappa index of agreement.

\begin{tabular}{c|c}
\hline \hline the Kappa index of agreement & interpretation \\
\hline \hline 0 & Poor \\
\hline $0.01-0.20$ & Slightly \\
\hline $0.21-0.40$ & Considerable \\
\hline $0.41-0.60$ & Moderate \\
\hline $0.61-0.80$ & Substantial \\
\hline $0.81-1.00$ & Excellent \\
\hline \hline
\end{tabular}

The results demonstrate the excellent performance of human skin pixel-based segmentation in the HSM color space proposed in this work when compared with the moderate Kappa index of agreement of the $\mathrm{YCbCr}$ color space and the considerable Kappa index of agreement of the HSV color space. An overall precision of HSM color space exceeds 14.9 and 24.8 on the $\mathrm{YCbCr}$ and HSV color spaces, respectively. In the Table 2 can be verified the interpretation of the Kappa index of agreement.

In the pixel-bsed segmentation of the trumpet flower tree, it was verified that the MDL when used in HSV color space failed to segment any type of flower, when it was applied in $\mathrm{YCbCr}$ color space it was segmented only the golden trumpet flower tree and angel trumpet flower tree, but when it was applied in HSM color space it was segmented the three types of trumpet flower tree (pink, golden and angel). The Table 3 demonstrates the excellent performance of the HSM color space when compared with the moderate Kappa index of agreement of the $\mathrm{YCbCr}$ color space and the poor Kappa index of agreement of the HSV color space in the pixel-based segmentation of trumpet flower tree.

To validate the performance of the face sketch image, a face sketch test database composed of images with differences in facial expressions of the AR database was set up. The face sketch databse was composed of 95 classes, where each class contained 4 images, so the database had 380 images. It was developed a CBIR (Content-Based Image Retrieval) [15] 


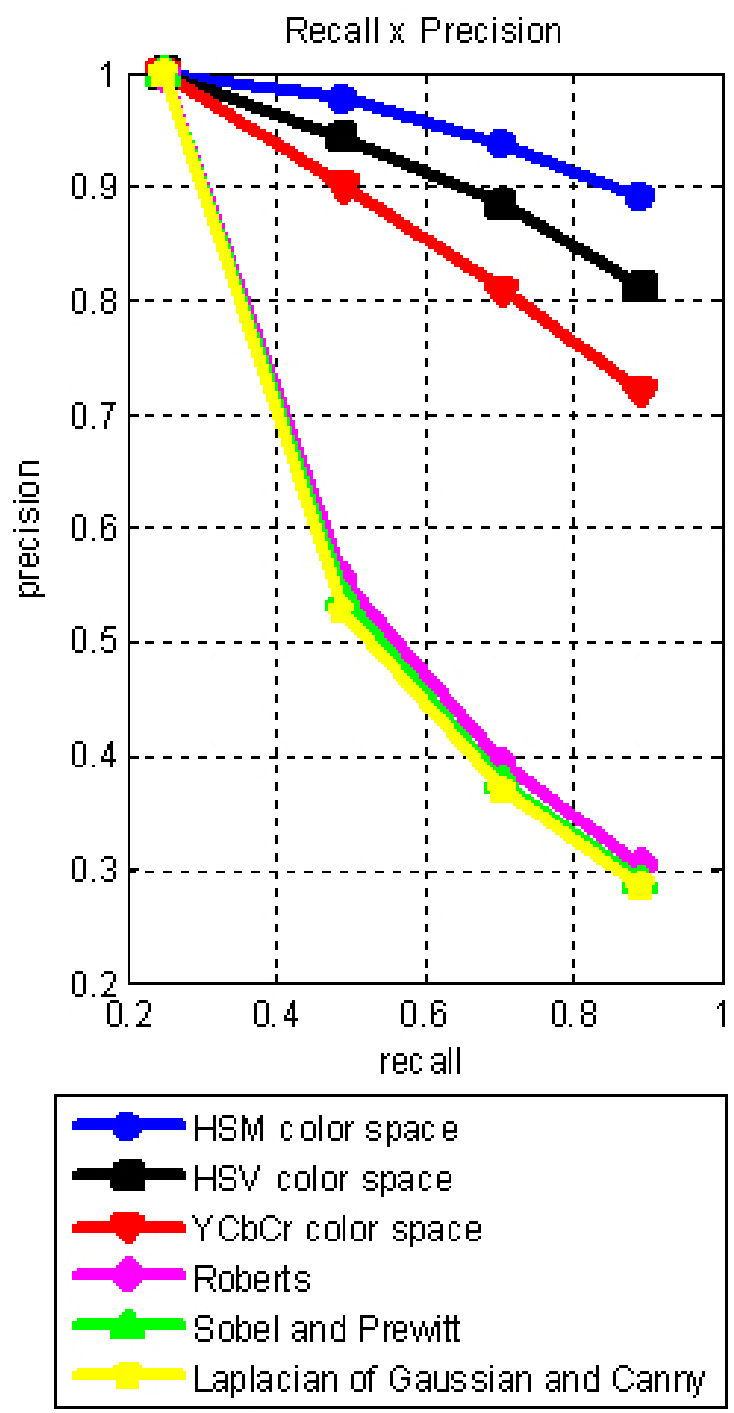

Figura 6. RP curves for the face sketch images and edge detectors. 
HSM: A New Color Space used in the Processing of Color Images

Tabela 3. Performance index of the trumpet flower tree.

\begin{tabular}{l||c|c|c}
\hline \hline Color Spaces & HSM & HSV & YCbCr \\
\hline trumpet flower classification rate & $99.8 \%$ & $99.7 \%$ & $64.6 \%$ \\
\hline non-trumpet flower classification rate & $93.4 \%$ & $0.36 \%$ & $80.3 \%$ \\
\hline Overall precision & $97.5 \%$ & $64.5 \%$ & $70.1 \%$ \\
\hline Kappa index of agreement & 0.95 & 0.00 & 0.41 \\
\hline \hline
\end{tabular}

system, that it used PCA (Principal Components Analysis)[1] to define the feature vector for the overall description of the database image. To define the projection matrix of the PCA subspace, each database image with spatial resolution $576 \times 768 \times 3$ pixels was converted to the grayscale spatial resolution with $576 \times 768$ pixels, and subsequently, the grayscale image was reduced to spatial resolution of $32 \times 36$ pixels and then, it was converted into a vector with $1152(32 \times 36)$ pixels. After, all vectors were grouped together to define a matrix with dimension $1152 \times 380$ and it was calculated the covariance matrix. Finally, the eigenvalues and eigenvectors matrix were calculated. The projection matrix of the PCA subspace is defined by the columns of the eigenvalues matrix corresponding to the columns of the 100 largest eigenvectors matrix. The feature vector for the overall description of the each database image was defined by the transformation of the image to the grayscale and for low spatial resolution of $32 \times 36$ pixels represented by a vector. This vector image with 1152 pixels was multiplied by the projection matrix of PCA resulting in a vector of 100 values. It was applied the Euclidean distance in the all feature vector to verify the similarity of the database images in the CBIR system to calculated the RP values. It was used the sequential access to calculated the average RP values of the all database images for the search of the first, the second, the third and fourth image closer to query image. Figure 6 shows the RP curves (recall x precision) [2] of the database images. The curves illustrate the performance of the images acquired by the HSM color space in relation to the HSV and the $\mathrm{YCbCr}$ color spaces and the edge detectors of Sobel, Prewitt, Roberts, Laplacian of Gaussian and Canny methods.

\section{Conclusions}

This paper proposed a new approach for interpreting digital color images. The color mixture is inspired by the technique that artists employ to create an oil painting by overlapping layers of different shades, as well as by the biological characteristics of the distribution of cones in the human retina as an interpreter of this mixture. The proposed methodology indicates the mixture quantified by the binary weight of each color defined in the RGB color space, and generates planes that intersect the RGB color space, thereby creating the HSM 
color space. The MDL based on the difference in luminance between the image of any color space and the RGB was implemented. When the MDL was applied in the HSM color space, its performance was superior to the other color spaces (HSV and $\mathrm{YCbCr}$ ), demonstrating not only the high efficiency of the new color space for the pixel-based segmentation of human skin, the pixel-based segmentation of trumpet flower tree and the face sketches but also the simplicity of its implementation.

\section{Referências}

[1] Baek, K.;Bartlett, M. S.;Draper, B. A. Recognizing faces with pca and ica. In Computer Vision and Image Understand, volume 91, pages 115-137, 2003.

[2] Baeza-Yates, R.;Ribeiro Neto, B. Modern Information Retrieval. ACM Press, New York, 1999.

[3] Bishop, Y.;Fienberg, S.;Holland, P. Discrete multivariate analysis: theory and practice. MIT, Cambridge, USA, 1975.

[4] Carroll, J.;Neitz, M.;Neitz, J. Estimates of 1:m cone ratio from erg flicker photometry and genetics. In Journal Vision, volume 2, pages 531-542, 2002.

[5] Gonzalez, R. C.;Woods, R. E.;Eddins, S. L. Digital Image Processing using Matlab. Pearson Education, 2004.

[6] Gunther, K. L.;Dobkins, K. R. Individual differences in chromatic (red/green) contrast sensitivity are constrained by the relative number of 1 - versus $\mathrm{m}$-cones in the eye. In Vision Research, volume 42, pages 1367-1378, May 2002.

[7] Hofer, H.;Carroll, J.;Neitz, J.;Neitz, M.;Williams, D. R. Organization of the human trichromatic cone mosaic. In The Journal of Neuroscience, volume 25, pages 96699679, October 2005.

[8] Kakumanu, P.;Makrogiannis, S.;Bourbakis, N. A survey of skin-color modeling and detection methods. In Pattern Recognition, volume 40, pages 1106-1122, March 2007.

[9] Maltoni, D.;et al. Handbook of Fingerprint Recognition. Springer, 2003.

[10] Martinez, A. M.;Benavente, R. The ar face database. Technical report, Purdue University, June 1998. http://cobweb.ecn.purdue.edu/ aleix/ aleix_face_DB.html accessed in March 2009.

[11] Morris, T. Multimedia Systems: Delivering, Generating, and Interacting with Multimedia. Springer, 2000. 
[12] Phillips, P. J.;Moon, H.;Rauss, P. J.;Rizvi, S. The feret evaluation methodology for face recognition algorithms. In IEEE Transactions on Pattern Analysis and Machine Intelligence, volume 22, pages 1090-1104, October 2000.

[13] Sebe, N.;Cohen, T.;Huang, T. S.;Gevers, T. Skin detection, a bayesian network approach. In Proceedings of the 17th International Conference on Pattern Recognition (ICPRO4), volume 2, pages 903-906, August 2004.

[14] Severino Jr, O. Colors mixture: A new approach for color processing and its application in image segmentation. Master's thesis, Engineering School of São Carlos of the São Paulo University, São Carlos, May 2009.

[15] Smeulders, A. W. M. et al. Content-based image retrieval at the end of the early years. In IEEE Transactions on Pattern Analysis and Machine Intelligence, volume 22, pages 1349-1380, 2000.

[16] Soriano, M.;Martinkauppi, J. B.;Huovinen, S.;Lääksonen, M. Adaptive skin color modeling using the skin locus for selecting training pixels. In Pattern Recognition, volume 36, pages 681-690, March 2003.

[17] Störring, M.;Koèka, T.;Anderson, H. J.;Granum, E. Tracking regions of human skin through illumination changes. In Pattern Recognition Letters, volume 24, pages 17151723, Jully 2003. 